\title{
Los caminos de la innovación y la inclusión en las empresas multinacionales que operan en México
}

JORGE GARRILO", REDI GOMI" GRACIELA BENSUSÁN"*

\section{Resumen}

Innovación e inclusión son dos temas centrales en la agenda pública en México. En los últimos años, las políticas públicas han estado encaminadas a incentivar la innovación a través de diversos programas, con la aspiración de que esta a su vez incentive la inclusión social. Por su parte, en el ámbito académico, las discusiones teóricas actuales han analizado la relación de estos dos conceptos, desde diferentes perspectivas. En este sentido, las Empresas Multinacionales (EMN) son actores clave para observar los procesos de innovación e inclusión, dado que permiten estudiar ambos fenómenos. Con base en los resultados de la encuesta sobre EMN, representativa a nivel nacional, efectuada a una muestra de 171 firmas multinacionales en México, entre 2008 y 2009, e información del Ranking Nacional de Ciencia, Tecnología e Innovación, el presente artículo analiza el comportamiento de la innovación y la inclusión dentro de las EMN que operan en México, y la posible relación entre estas dos variables, así como la influencia del entorno institucional y económico sobre las mismas. Los principales resultados confirman una fuerte relación entre la inclusión e innovación en las EMN establecidas en el país, sin embargo, el panorama no es muy alentador, dado que poco menos de la cuarta parte de las empresas analizadas eran innovadoras e incluyentes a la vez. Lo que indica que los caminos de la innovación y la inclusión son caminos que aún no han logrado equipararse.

Palabras claves: Innovación. Inclusión. Empresas Multinacionales. México.

\footnotetext{
* El Colegio de la Frontera Norte, México

${ }^{* *}$ Universidad Autónoma Metropolitana, México
} 


\title{
The paths of innovation and inclusion in multinational enterprises operating in Mexico
}

\begin{abstract}
Innovation and inclusion are two key topics in the public agenda in Mexico. In recent years, public policies have focused on the promotion of innovation through various programs, expecting that this, in turn, might encourage social inclusion. In the academic field, current theoretical discussions have analyzed the relation between these two concepts from different perspectives. In this sense, Multinational Enterprises (MNE) are key actors for observing the processes of innovation and social inclusion, since they allow to study both phenomena. Based on the results of a nationally representative survey on MNEs carried out in Mexico, between 2008 and 2009, comprising a sample of 171 multinational fir$\mathrm{ms}$, and on information from the National Ranking of Science, Technology and Innovation, this article presents the analysis of the behavior of Innovation and inclusion within MNEs operating in Mexico, and the possible relationship between these two variables, as well as the influence of the institutional and economic environment over them. The main results confirm a strong relationship between inclusion and innovation in MNEs established in the country. However, the picture is not very encouraging, since less than a quarter of the companies analyzed was simultaneously innovative and inclusive. This indicates that the paths of innovation and inclusion have not yet matched.
\end{abstract}

Keywords: Innovation. Inclusion. Multinational Corporations. Mexico. 


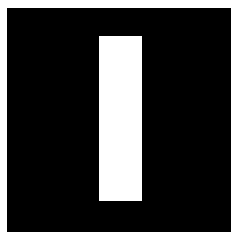

nnovar es un concepto que despierta hoy en día cierto fervor y sobre el cual empresas, gobiernos e instituciones sociales cifran expectativas por un supuesto o real impacto sobre la eficiencia de los procesos, la competitividad y el desarrollo económico. Aunque con otras connotaciones, incluso también en el ámbito académico es visible esta misma apuesta. La frase "innovar o morir" es frecuentemente utilizada como una guía máxima que, si bien es normativa en señalar el camino que debería seguirse, se mueve siempre dentro de los límites de lo técnico y lo pragmático. Es decir, con una connotación, digamos, más neutral. No cumplir con estos lineamientos no convierte a sus "infractores" en objeto de desaprobación o condena alguna. Pero existen en cambio mecanismos para incentivar y apoyar financieramente la actividad. La inclusión, por el contrario, constituye en gran medida un valor moral, político y social a favor de la equidad y la diversidad. Con frecuencia se mueve en los terrenos cambiantes del derecho y de la justicia social. Por eso, aún dentro de la academia, las discusiones sobre este tema pueden llevar consigo una fuerte carga emocional. Contribuir a la exclusión de personas, grupos, territorios, ya sea sin premeditación (como resultado de las lógicas económicas) o intencionalmente (algo cercano a la discriminación), puede generar críticas de diverso tipo e intensidad para los responsables. Pero para promover y estimular la inclusión no existen, como ocurre en el caso de la innovación, incentivos materiales: tan solo se adjudican distinciones y reconocimientos para quienes logran avances perceptibles en dirección a esa meta.

Este trabajo persigue varios propósitos. El primero es examinar cómo se comportan la innovación y la inclusión dentro de las EMN (empresas multinacionales) que operan en México. Asumiendo para ambos procesos una connotación positiva - en un caso, porque contribuye al desarrollo, en el otro, porque es justo - , lo ideal sería entonces que las EMN 
se encuentren en la "vía alta" y desplieguen a plenitud tanto la innovación como la inclusión. El peor escenario es que no se presente ninguna de las dos. ¿Cuántas de las EMN se encuentran en cada una de estas dos situaciones — o de alguna condición intermedia — y qué propiedades las caracterizan? Un segundo propósito es tratar de determinar si entre estos dos fenómenos existe algún tipo de relación. Por lo que se planteó al inicio, parecieran completamente ajenos. Sin embargo, ya sea de manera directa o indirecta, en la literatura se establecen relaciones entre ambos. ¿Se puede fijar también algún tipo de entrelazamiento entre ambas en las EMN instaladas en México? Esta es una preocupación que se sostiene a lo largo de todo el trabajo y por eso se examinarán, por su importancia, las discusiones sobre el particular, tanto en los ejes macro y micro. Por último, un tercer propósito es explorar en qué medida tales procesos se encuentran influenciados por los entornos institucionales y económicos correspondientes a los sistemas territoriales de innovación.

Para apoyar el análisis empírico se contó fundamentalmente con los datos provenientes de una encuesta realizada entre 2008 y 2009 a una muestra representativa de 171 corporativos localizados a lo largo del país. Parte de los análisis que aquí se muestran habían sido previamente expuestos en un trabajo anterior (Carrillo; Gomis, 2014).

La estructura del documento es como sigue. Además de esta introducción, las ideas se van a exponer en siete apartados. En el primero, se presenta una breve discusión de la literatura acerca de la relación entre los conceptos de innovación e inclusión. En el segundo, se justifican los casos, es decir, por qué el estudio del fenómeno de interés en las EMN. En el tercero, se explican, también de manera breve, el origen, naturaleza y limitaciones de los datos. En el cuarto y quinto apartados, se realizan los análisis empíricos. Por último, en el sexto, se resumen las ideas y se presentan las conclusiones. 


\section{La interrelación innovación-inclusión: principales ejes de discusión}

La innovación y la inclusión aparecen como dos conceptos enlazados dentro de las discusiones teóricas actuales. La naturaleza y la lógica de esa conexión va a depender en gran medida del horizonte macro o micro desde el cual se establece el análisis. Es decir, si la mirada recae en los procesos que abarcan a grandes grupos sociales y a territorios amplios; o si, por el contrario, ese horizonte es más inmediato, circunscrito sólo a lo que ocurre dentro de los márgenes de una empresa.

Veamos primeramente dos enfoques pertenecientes al nivel macro, y de los cuales consideramos importante recuperar sus argumentos más relevantes. Uno, de corte más económico, orientado a las causas del desarrollo. El otro, de naturaleza más social, con la mirada puesta sobre las consecuencias del mismo, tal y como ha tenido lugar hasta ahora.

La lógica de las ideas del primero de estos dos enfoques macro es presentada por Lee (2014). Su interés es explicar el punto de vista personal acerca de cómo superar lo que Gill y Kharas (2007) conceptualizaron como trampa del ingreso medio. La inclusión es concebida como sinónimo de crecimiento incluyente, a partir de una esperada distribución más equitativa de los frutos del crecimiento económico, una situación que supuestamente derivaría de la elevación general de los salarios. Un problema central es que no todas las formas de crecimiento y desarrollo alcanzan este resultado, de lo que abundan ejemplos, sobre todo entre los países subdesarrollados. Lee parte de considerar que la relación entre ambas variables — crecimiento e inclusión - sigue una trayectoria en forma de " $U$ " invertida, similar al de la curva de Kuznets, de manera tal que "la desigualdad del ingreso aumenta con el tiempo, a medida que el país se desarrolla; pero que una vez que el país alcanza un nivel de ingresos determinado, la desigualdad del ingreso comienza a disminuir" 
(Lee, 2014, p. 219). De acuerdo a su razonamiento, para que pueda lograrse la relación virtuosa entre desarrollo e inclusión es un requisito imprescindible que tal crecimiento sea "sostenido" en el tiempo. Y aquí es que entra en juego en la ecuación de Lee la innovación. Es uno de los elementos esenciales que abonaría para que el crecimiento pueda perdurar por períodos más o menos largos hasta permitir que la curva alcance el hipotético punto de giro a partir del cual las desigualdades comenzarían finalmente a reducirse. La impronta que Lee le atribuye a la innovación sobre la inclusión es crucial, al extremo de suponer que en su abandono se encontraría justamente el fundamento de la trampa del ingreso medio.

Tres elementos que se desprenden de lo planteado en esta perspectiva merecen destacarse. Uno es que el vínculo se asume en un sólo sentido. La inclusión sería siempre un resultado o efecto de la influencia de la innovación, no al revés. Pero tal influencia - y este es otro elemento a destacar - no sería directa, sino indirecta y mediata. La función que cumple la innovación en el esquema explicativo es contribuir a prolongar el crecimiento. Pero es este último, y no la innovación en sí misma, lo que en definitiva favorecería la inclusión. Y, por último, un tercer elemento es que la innovación termina siendo concebida como un fenómeno indiferenciado con impactos uniformes. No se distinguen tipos o formas de innovación, tal y como sí ocurre respecto del crecimiento. Basta su presencia para suponer una extensión del crecimiento y con ello empezar a disminuir en algún momento posterior las desigualdades.

Para esbozar el segundo enfoque macro al que aludiremos en este trabajo servirá de apoyo lo expuesto, entre otros, por Papaioannou (2014), Foster y Heeks (2013) y la OECD (2013a). En el corazón de sus preocupaciones, se encuentra el concepto de "innovación incluyente", que en esencia se refiere a aquellas formas de innovación que deliberadamente incorporan o buscan incorporar de alguna manera las necesida- 
des de aquellos grupos sociales marginados y excluidos sistemáticamente de los procesos de desarrollo, especialmente los de más bajos ingresos (OECD, 2013a). La raíz de esta preocupación radica en que se asume que las innovaciones tecnológicas en el sector formal han fracasado hasta ahora tanto en comprender como en solucionar los problemas de exclusión social (Papaioannou, 2014). Entre otras razones, porque siempre han sido concebidas en función de las necesidades de las empresas, de su eficiencia y competitividad, y no con propósitos específicos de inclusión. De acuerdo al propio Papaioannou, para que un modelo de innovación pueda ser calificado como incluyente, es preciso que haya evidencia de que, en los contextos específicos de desarrollo en los que se despliega, persigue la satisfacción de las necesidades humanas básicas sobre una base de equidad. La inclusión es concebida como una cuestión de elemental justicia global (Papaioannou, 2014).

Al contrastar este segundo enfoque macro con el propuesto por Lee (2014), se puede entender mejor la naturaleza de la interrelación teórica de la innovación y la inclusión que en el mismo se formula. Entre ambas existen varios elementos semejantes, pero también aspectos divergentes: 1. La innovación adquiere un papel más preponderante con respecto a la inclusión. También la influencia viaja en un solo sentido. Pero no actúa de forma mediata, a través del crecimiento y del desarrollo, sino directamente. La inclusión es un objetivo expresamente buscado a través de la innovación.

2. La innovación deja de ser indiferenciada y se convierte en un fenómeno de múltiples aristas. Foster y Heeks (2013, p. 335) destacan cuatro aspectos de la innovación - importantes en relación con la inclusión -, que han sido enfatizados por la literatura: (a) la concepción o previsión acerca de la relevancia que podría tener la innovación para los excluidos; (b) la producción o incorporación de los excluidos en el 
proceso mismo de desarrollo de nuevos productos y servicios, (c) el consumo o las capacidades de absorción que poseen los excluidos para adoptar las innovaciones; (d) el impacto que las innovaciones tienen sobre los medios de vida de los excluidos. El segundo de estos cuatro aspectos tiene gran relevancia para este trabajo, en la medida en que, como se verá más adelante, lo que nos interesa desde la perspectiva de la empresa tiene que ver fundamentalmente con el proceso de "producción" de la innovación, una de cuyas dimensiones es precisamente quienes participan en ese proceso.

La relación entre innovación e inclusión adquiere, en la perspectiva micro, matices diferentes a los examinados para el nivel macro. Aquí hay varios puntos a enfatizar:

1. Lo que se tiene sobre todo presente en este nivel no son los efectos externos y generales de la actividad innovadora de la empresa, sino que el interés está más bien referido a las prácticas y procesos que caracterizan su dinámica interna en lo referente a la innovación y a la inclusión;

2. La inclusión no equivale a la reducción de las desigualdades sociales globales, sino a la atención específica de las diversidades humanas mediante su incorporación a los procesos productivos. Aunque de alguna manera relacionado, esta forma de entender la inclusión sería un concepto de alcance más limitado que el manejado por los otros enfoques, de alguna manera más afines al de progreso social. Por eso, la inclusión a nivel de empresas se convierte fundamentalmente en un valor. $Y$, en este sentido, existe en la actualidad un amplio movimiento de interés político y social que distingue y valora como incluyentes a las empresas que contratan a personas con alguna discapacidad, observando, al mismo tiempo, que en el procedimiento se cumplan todas las disposiciones que al respecto marca la ley. En un trabajo anterior de Carrillo y De los Santos (2014), se describen algunas medidas de inclusión social 
en México, como condiciones de empleo y trabajo en multinacionales, entre las que destacan ejemplos de mejores prácticas sostenibles, tanto públicas como privadas (Great Place to Work, "Programa Calidad Integral y Modernización", "Iniciativa de Empleos Verdes"); proyectos de responsabilidad social (de CONEVyT [Consejo Nacional de Educación para la Vida y el Trabajo] y de INEA [Instituto Nacional para la Educación de los Adultos]); regulaciones ("Ley de Fomento a la Competitividad y Desarrollo Económico" y "Foro Mujer Pyme"); y becas privadas (como las "Becas Samsung" a estudiantes destacados). Se hace énfasis sobre el reclutamiento de los discapacitados (OIT, 2015), aunque igualmente incluye otros grupos sociales.

3. La relación entre innovación e inclusión se expresa en un doble sentido de dirección. La innovación, pensada en función de las necesidades de los excluidos, puede llevar a que los mismos participen en los procesos productivos. Esto tiene que ver con lo planteado por Foster y Heeks (2013), examinado antes, en el sentido de concebir la inclusión como parte del innovation proccess. La influencia va desde la innovación hacia la inclusión. Pero la relación a la inversa es igualmente enfatizada. En este sentido, lo que Page (2007) Ilama la lógica de la diversidad es que las organizaciones con diversidad de empleados suelen tener mejor desempeño (p. 6). En mayo del 2014, uno de los vicepresidentes de Google publicó una autocrítica en el blog oficial de la empresa. Con datos concretos sobre la composición étnica y por sexo, admite que se encuentran lejos de lo que aspiran en relación con la diversidad de la fuerza laboral. Porque, a su entender, y es lo que se quiere subrayar aquí, las diferencias generan un trabajo más innovador (Bock, 2014).

4. Algo importante a destacar tiene que ver con los ámbitos espaciales a los que se integran los procesos de innovación de las empresas. Si bien buena parte de esta se registra en la empresa, no siempre el impulso 
de tales innovaciones se genera en su interior. Ya a mitad de los años ochenta, Drucker (1993) describía dos tipos de fuentes principales de oportunidad innovadora: fuentes de dentro de la empresa y fuentes de fuera de la empresa. Hoy en día, la idea de que fundamentalmente los procesos y desarrollos asociados a innovación surgen y progresan al amparo de un entorno que los asiste y moldea, goza de amplia aceptación. Conceptos como Milieux Innovateurs (Aydalot, 1986), Learning regions (Florida, 1995), Intelligent regions (Cooke; Morgan, 1991), entre otros, dan cuenta del grado de penetración que la idea de un entorno innovador ha ido adquiriendo en la literatura especializada. En todos los casos, el centro de gravedad del interés y la explicación se desplazan desde el nivel del empresario innovador, o de la firma innovadora, al del contexto, entendido como una suerte de ecosistema de innovación. La noción de fondo es que la empresa no opera de manera aislada, sino permanentemente vinculada con otros actores en sistemas de redes sociales. Este es otro aspecto que se quiere examinar de manera concreta en el estudio.

Los casos de estudio: las empresas multinacionales en México

¿Por qué las EMN como casos de estudio? ¿Por qué buscar examinar relación entre innovación e inclusión específicamente en las EMN? En las discusiones sobre desarrollo e innovación es siempre importante tener presente a las EMN. Son actores clave en esos procesos, por lo que autores como Kristensen y Zeitlin (2005) consideran a las multinacionales como un laboratorio mundial para la innovación.

Las empresas multinacionales en México son, en general, muy dinámicas, pero la situación es muy variada según el indicador que se revise. Indicadores relevantes son el cambio en el empleo, la subcontratación y 
el número de establecimientos que mantiene cada multinacional (Carrillo, 2013). No obstante lo anterior, y pese a la enorme importancia económica y social que representan las empresas multinacionales en México, tanto extranjeras como domésticas, y al papel en la innovación y la inclusión social, existen pocos estudios en este país y en su mayoría se han enfocado en la manufactura (Carrillo, 2012).

Cypher y Dietz (2009) afirman que, hoy en día, las multinacionales se encuentran en la mayoría de las naciones menos desarrolladas. Para el desarrollo nacional, el indicador más revelador de la incorporación exitosa de la transferencia de conocimientos y la tecnología se relaciona con la magnitud de los vínculos nacionales entre las EMN y la economía receptora. Como ejemplo teórico, una multinacional con una estructura compleja cuya actividad principal es la manufactura puede estar conformada de varias empresas, plantas productivas, empresas de servicios, centros técnicos y de un corporativo central y otros regionales. Mientras que una multinacional de servicios puede estar conformada por varias sucursales, empresas, una planta productiva y varios corporativos (regionales y centrales) (Carrillo, 2013).

Dunning y Lundan (2008) afirman que existen varios criterios para evaluar el grado o intensidad de las empresas multinacionales. Entre ellos, destacan: a) el número y tamaño de las filiales extranjeras o empresas asociadas que posee o ejerce control; b) el número de países en los que es propietaria o de alguna manera controla las actividades de mayor valor agregado; c) la proporción de activos globales, ingresos y empleo contabilizada por sus filiales extranjeras; d) el grado en que la administración y propiedad se internacionalizan; e) la medida en que sus actividades de mayor valor, por ejemplo, la investigación y desarrollo, están internacionalizadas, ya que esta medida tiene la intención de capturar la calidad o la profundidad de la producción extranjera, y la contribución de las filiales 
extranjeras a acceder o crear directamente nuevos conocimientos; f) el grado de las ventajas sistémicas derivadas de su gobierno y la influencia sobre una red de actividades económicas ubicadas en distintos países; g) y, por último, la toma de decisiones en relación con asuntos financieros y de comercialización, que se delegan en las filiales extranjeras.

La declaración tripartita de principios sobre las empresas multinacionales y la política social, adoptada por el Consejo de Administración de la Oficina Internacional del Trabajo, declara que

Las empresas multinacionales desempeñan un papel muy importante en las economías de la mayor parte de los países y en las relaciones económicas internacionales, que es de interés creciente para los gobiernos, así como para los empleadores, los trabajadores y sus respectivas organizaciones. Mediante las inversiones directas internacionales y otros medios, estas empresas pueden aportar ventajas substanciales al país de acogida y los países de origen, contribuyendo a una utilización más eficaz del capital, la tecnología y el trabajo (OIT, 2006, p. 1).

Por otra parte, la Organización para la Cooperación y el Desarrollo Económico (OECD, 2013b) afirma que las empresas multinacionales deben contribuir al progreso económico, social y medio ambiental, con el objetivo de lograr un desarrollo sostenible.

Dunning (1993) resumió gran parte de la teoría económica de las multinacionales en los tres factores básicos: 1) Ventajas de propiedad (conocimiento, tecnología); 2) ubicación (de las filiales y la inversión extranjera directa); y 3) internalización (transferencia de ventajas dentro de la empresa). En la presente sección, se muestra un debate sobre el papel que juegan las corporaciones multinacionales dentro de los países en que se instalan, en este caso en México. 


\section{Los datos para el análisis empírico}

La información que va a servir de fuente primaria para este trabajo se originó en una encuesta efectuada a una muestra de 171 firmas multinacionales en México - en un universo de 922 - , entre fines del año 2008 y principios del 2009. La encuesta se produjo en el marco de un proyecto de investigación desarrollado en El Colegio de la Frontera Norte, con el objetivo de estudiar la estructura organizacional, la innovación y las prácticas de empleo de las empresas multinacionales en México. Este proyecto, a su vez, siguió de cerca los lineamientos establecidos por Intrepid $^{1}$ una red internacional de investigadores independientes que busca consolidar, con el aporte de todos los grupos nacionales participantes, una base de datos confiable y relativamente homogénea que permita producir análisis comparables de alta calidad sobre políticas y prácticas de empleo de las empresas multinacionales alrededor del mundo.

La población objetivo de la investigación resulta de la definición operativa adoptada de común acuerdo por los participantes en la agrupación Intrepid, según la cual se considerará como multinacionales a aquellas firmas que participan económicamente en por lo menos otro país además de México, y que tienen al menos 500 empleados en total, pero con un mínimo de 100 empleados en el país donde se origina la encuesta.

Es importante decir que los objetivos del proyecto para el cual fue levantada la encuesta no iban dirigidos al estudio de la inclusión. Por eso presentan limitaciones para los propósitos de este trabajo. Existe una enorme dificultad para obtener información empírica exhaustiva y fidedigna sobre las condiciones laborales en las EMN. No obstante, los datos

\footnotetext{
${ }^{1}$ Intrepid es el acrónimo de Investigation of Transnational Employment Practices: an International Database. En esta red, participan investigadores de nueve países: Inglaterra, Irlanda, España, Canadá, México, Argentina, Noruega, Finlandia, Australia. Sus miembros se reúnen aproximadamente cada dos años con el propósito de intercambiar información y trazar estrategias de investigación de largo aliento, enfatizando en los estudios comparados.
} 
de la encuesta permiten dar cuenta de las diferencias entre las empresas en el grado en que tienen lugar tanto los procesos de inclusión como los de innovación, aunque la precisión sobre estos fenómenos a partir de los mismos sea forzosamente limitada.

\section{La innovación y la inclusión en las EMN en México}

El objetivo primario en este estudio es determinar el comportamiento de las EMN en el eje combinado de la innovación y la inclusión, y consistiría esencialmente en examinar la distribución de las mismas dentro de la variable que pudiera denominarse como "grado de éxito en el desarrollo integrado de la innovación y la inclusión". La idea no es otra que tener una visión de conjunto del perfil concreto de las relaciones entre esas dos dimensiones para las EMN en México, a partir de precisar la manera específica en que las mismas se combinan en cada caso particular.

Por una parte, al considerar estas dimensiones como dicotómicas si innova o no, si es incluyente o no lo es - , en términos teóricos pueden distinguirse al menos tres situaciones posibles — o tipos ideales —: (1) las EMN son innovadoras y también incluyentes; (2) las EMN son innovadoras o incluyentes, pero no ambas cosas a la vez; (3) las EMN carecen por igual de las dos características. Por otra, se puede establecer una especie de ordenamiento escalonado de estos tres tipos, si aceptamos que desarrollar ya sean procesos de innovación o de inclusión — sólo uno de ellos, no ambos - , representaría una suerte de "grado" intermedio entre otras dos situaciones más extremas, una de grado inferior (carecer, de estas dos condiciones) y la otra de nivel superior (disponer de ambas a la vez). Así, los tres valores del grado de éxito en el binomio innovación-inclusión, correspondientes a los tres tipos de combinación presentados antes, serían, respectivamente: (a) éxito total; (b) éxito parcial, y; (c) sin éxito. 
Ahora bien, a fin de poder establecer esa variable combinada y lograr alcanzar el objetivo propuesto, resulta imperativo, como paso previo, las identificaciones por separado tanto de la condición innovadora como de la condición incluyente de las EMN. Para ello, se elaboraron dos índices específicos, uno para cada dimensión, a partir de los cuales se optó por dividir la dimensión en cuestión en dos valores; esto es, si tiene la cualidad en cuestión o bien carece de la misma. En términos operativos, ambos índices fueron elaborados a partir de un conjunto amplio de indicadores provenientes de la base de datos (véase cuadro 1). Los datos de la base son preexistentes. Y, desde luego, la misma no contendría todos los aspectos que pudieran considerarse relevantes para agotar el entendimiento cabal de la innovación o la inclusión. No obstante, se asume que las variables incluidas permiten una observación de las dos dimensiones acorde a los propósitos del estudio.

En cuanto a la innovación, tomamos como el elemento central el involucramiento de la empresa en las siguientes actividades consideradas innovadoras: contar con un departamento de I+D, reconocer que en la EMN se realiza innovación de reversa ${ }^{2}$, tener vinculación con centros educativos para desarrollos de I+D, y haber buscado apoyos del gobierno para el mismo fin. Si bien la información ofrecida no permite determinar realmente cuánta innovación real realiza la empresa, ni de qué tipo es la misma (en producto, procesos, etc.), no obstante, todos ellos son indicadores que apuntan en una misma dirección: hablan de una mayor o menor vocación innovadora de la empresa. Su consideración resulta importante bajo el supuesto de que, a la larga, esta vocación debe traducirse en innovaciones concretas.

\footnotetext{
${ }^{2}$ Innovación en reversa, significa realizar I+D en las operaciones de la multinacional en México que es utilizada por otras empresas de la misma multinacional en otros países. Es decir, transferencia de conocimiento a la inversa.
} 
Cuadro 1. Variables, indicadores, valores. Operacionalización de las variables "vocación innovadora" y "condición de inclusión" de las EMN en México

\begin{tabular}{|c|c|c|}
\hline Variable & Indicadores & Valores \\
\hline $\begin{array}{l}\text { Vocación } \\
\text { innovadora }\end{array}$ & $\begin{array}{l}\text {-Empleo en investigación y desarrollo (I+D) } \\
\text {-Vinculación con universidades para desarrollo de } \\
\text { I+D } \\
\text {-Búsqueda de apoyos gobierno para desarrollo de } \\
\text { I+D } \\
\text { - Existencia de innovación de reversa }\end{array}$ & $\begin{array}{l}\bullet \text { Vocación } \\
\text { menos } \\
\text { marcada } \\
\text { •Vocación más } \\
\text { acentuada }\end{array}$ \\
\hline $\begin{array}{l}\text { Condición } \\
\text { de inclusión }\end{array}$ & $\begin{array}{l}\bullet \% \text { de mujeres } \\
\bullet \% \text { de trabajadores temporales } \\
\text { • Grado de monitoreo por parte de sus directivos } \\
\text { sobre la diversidad de la mano de obra } \\
\text { •Si se favorece la movilidad ascendente } \\
\text { - Si se privilegia a los gerentes locales sobre los } \\
\text { provenientes del país de origen } \\
\text { - Nivel de apoyo, comunicación e intercambio entre } \\
\text { los trabajadores y los gerentes } \\
\text { - Reconocimiento de la representación de los } \\
\text { trabajadores } \\
\text {-Vigilancia de los derechos de los trabajadores en las } \\
\text { empresas proveedoras }\end{array}$ & $\begin{array}{l}\text {-Más incluyente } \\
\text { •Menos } \\
\text { incluyente }\end{array}$ \\
\hline
\end{tabular}

Fuente: Elaboración de los autores

Por su parte, el carácter incluyente de las empresas se tomó en un sentido más exhaustivo e integral, que considerara no sólo el factor diversidad. Se buscó determinar cuáles empresas tienen mayor disposición que otras para beneficiar no sólo la participación de grupos en situación de vulnerabilidad, sino también incrementar las oportunidades de desarrollo y, en general, favorecer las condiciones laborales de todos sus trabajadores. Se utilizaron igualmente varias variables de la base para construir un indicador que apuntara en este mismo sentido. Y así, a partir de estas consideraciones, definimos operacionalmente como empresa incluyente a aquella que tiende a emplear mayor proporción de mujeres; a tener menor proporción de trabajadores temporales; a mantener un mayor 
monitoreo por parte de sus directivos sobre la diversidad de la mano de obra; a favorecer las carreras internas (movilidad ascendente); a privilegiar a los gerentes locales sobre los provenientes del país de origen de la EMN; a apoyar ampliamente formas de comunicación e intercambio entre los trabajadores y los gerentes; a reconocer la representación de los trabajadores; y, por último, a vigilar los derechos de los trabajadores en las empresas proveedoras. Esto es lo que se tiene entonces como referente conceptual al hablar en el trabajo de inclusión y de la condición más o menos incluyente de las empresas.

Resulta difícil imaginar en términos lógicos que una EMN legalmente establecida sea completamente excluyente. O que no realice, ni siquiera mínimamente, actividades de carácter innovador. Sin embargo, para facilitar el análisis de la información, las dos dimensiones se concibieron como binarias, tomando como punto de corte o separación de los dos valores contenidos el promedio en cada uno de los índices construidos. Por encima o por debajo de ese valor de corte fue establecida entonces la "presencia" o "ausencia" de la propiedad en cuestión. O, lo que es quizá, conceptualmente hablando, más cercano a la realidad: la mayor o menor presencia de las mismas. Veamos a continuación lo que nos arroja el análisis.

En el cuadro 2 se expone la distribución cruzada de las dos variables proyectadas. El resultado constituye en realidad la especificación concreta para el caso de las EMN en México de las tipologías presentadas más arriba correspondiente a los valores de la variable grado de éxito en la incorporación combinada de procesos de innovación e inclusión, y cuyas proporciones son las siguientes: (a) empresas de éxito total, incluyentes e innovadoras (21.5\%); (b) empresas de éxito parcial, incluyentes o innovadoras (33.4\%), y; (c) empresas sin éxito, ni incluyentes ni innovadoras (45.2\%). 
Cuadro 2. Empresas Multinacionales en México incluyentes e innovadoras

\begin{tabular}{|c|c|c|c|}
\hline \multirow{2}{*}{ Incluyente } & \multicolumn{2}{|c|}{ Innovadora } & \multirow{2}{*}{ Total } \\
\cline { 2 - 3 } & $\mathrm{Si}$ & No & \\
\hline $\mathrm{Si}$ & $21.5 \%$ & $27.2 \%$ & $48.6 \%$ \\
\hline $\mathrm{No}$ & $6.2 \%$ & $45.2 \%$ & $51.4 \%$ \\
\hline Total & $28 \%$ & $72 \%$ & $100 \%$ \\
\hline
\end{tabular}

Fuente: Elaboración de los autores. Encuesta de Corporaciones Multinacionales, Proyecto COLEF-CONACYT \# 55108, "Firmas Multinacionales en México: un estudio sobre la estructura organizacional, la innovación y las prácticas de empleo", 2008-2009.

Hay al menos tres ideas que emergen de la aproximación a la información recogida en el cuadro 2.

1. La primera es que tanto la propensión innovadora como el auspicio de medidas incluyentes son cualidades realmente excepcionales entre las EMN. Casi la mitad (45.2\%) carece simultáneamente de las mismas.

2. La segunda es que, examinadas por separado, la frecuencia de una y otra no ocurre en igual magnitud. Es relativamente más difícil encontrar a una empresa innovadora que a una incluyente. Aunque una parte significativa de las EMN no son incluyentes, la proporción de las que se encuentran en esta situación asciende a poco más de la mitad (51.4\%). Pero si lo que se considera es la innovación, entonces la proporción de las que no realizan esta actividad asciende a las tres cuartas partes (72\%). Ello significa que el "éxito parcial" viene sobre todo determinado por EMN incluyentes que no son innovadoras.

3. Por último, la tercera tiene que ver con el vínculo entre las dos variables. Como derivación de las ideas anteriores, se puede suponer que la innovación es el elemento más determinante entre los dos. La probabilidad de que una EMN sea incluyente, pero no innovadora, es alta (56\%). Pero al revés se invierte la situación. Es decir, si una EMN es innovadora, la probabilidad de que también sea incluyente se eleva al $77 \%$. 
Cabría preguntarse si el comportamiento general de las EMN en cuanto a la innovación y a la inclusión es similar en todas las circunstancias o si, por el contrario, sufre variaciones cuando cambian las condiciones bajo las cuales transcurren esos procesos. Considerando, en primer lugar, que la heterogeneidad es parte estructural de la caracterización del entorno industrial en México y, además, que la propensión innovadora puede ser el factor catalizador de los procesos de inclusión, en lo que sigue se tratará de determinar en qué medida la probabilidad de pertenecer a uno de estos grupos de "éxito" se ve influenciada por el contexto científico, técnico e innovador del territorio en el cual la EMN se encuentra enclavada. $\mathrm{Y}$ para ello es un requisito examinar primero la naturaleza de esos contextos en México.

\section{Ciencia, tecnología e innovación en las entidades federativas}

Otro de los objetivos primarios del estudio es la construcción de la variable "nivel de ciencia, tecnología e innovación". La misma está basada en la información recogida en el "Ranking Nacional de Ciencia, Tecnología e Innovación", publicado por el Foro Consultivo Científico y Tecnológico (Dutrénit \& Zúñiga-Bello, 2014). Se trata de un índice o medida resumen que integra información de diez dimensiones, y que sintéticamente da a conocer y describe las fortalezas, oportunidades y debilidades en CTI (Ciencia, Tecnología e Innovación) de cada una de las entidades federativas mexicanas, permitiendo adicionalmente el beneficio de enlistarlas en orden descendente.

Atendiendo al ordenamiento resultante, se consideraron tres niveles de CTI: alto, mediano y bajo. En el nivel alto, se encuentran las cinco entidades que, según el propio informe, "sobresalen en el ordenamiento" (Dutrénit; Zúñiga-Bello, 2014, p. 43). Estas son Distrito Federal, Nuevo 
León, Querétaro, Jalisco y Morelos. En el nivel intermedio, se encuentran aquellas entidades que, sin alcanzar la importancia de las cinco entidades primeras, tienen el valor del índice por encima de cero. Son entidades que se distinguen "porque en algunas de las dimensiones que mide el ranking han realizado esfuerzos significativos en la dinámica de sus sistemas de CTI" (Dutrénit; Zúñiga-Bello, 2014, p. 43). Sin realizar un listado exhaustivo, entre estas aparecen Puebla, Guanajuato, San Luis Potosí, Sinaloa, Tamaulipas, Baja California, Sur, Yucatán, Colima, Coahuila, Aguascalientes, Baja California, Chihuahua y Sonora. Por último, en el nivel más bajo, se ubican las restantes entidades, y que en el índice en el que se basa el ranking obtuvieron un valor por debajo de cero. En el extremo inferior de este tercer grupo de estados estarían Tabasco, Chiapas, Oaxaca, Guerrero y Campeche.

La gráfica 1 ilustra cómo se encuentran distribuidas las EMN en México en estos tres diferentes ecosistemas de innovación de acuerdo a la fuente consultada. Resulta obvio el vínculo entre el nivel de CTI de las entidades federativas y la localización de los corporativos de las EMN. Como se aprecia en la gráfica, más de la mitad (57\%) de las EMN de la base de datos están localizadas en estados con altos niveles de CTI. En los estados con niveles intermedios de CTI, se ubica sólo un poco más de la tercera parte (37.3\%) de las EMN. Y en entidades con niveles más bajos de $\mathrm{CTI}$, apenas se encuentra el $11.7 \%$ de estas empresas. La distribución es clara. Con los niveles de CTI aumenta también la concentración numérica de EMN. 
Gráfico 1. Distribución porcentual de las EMN en México de acuerdo al "nivel de CTI en la entidad de localización"

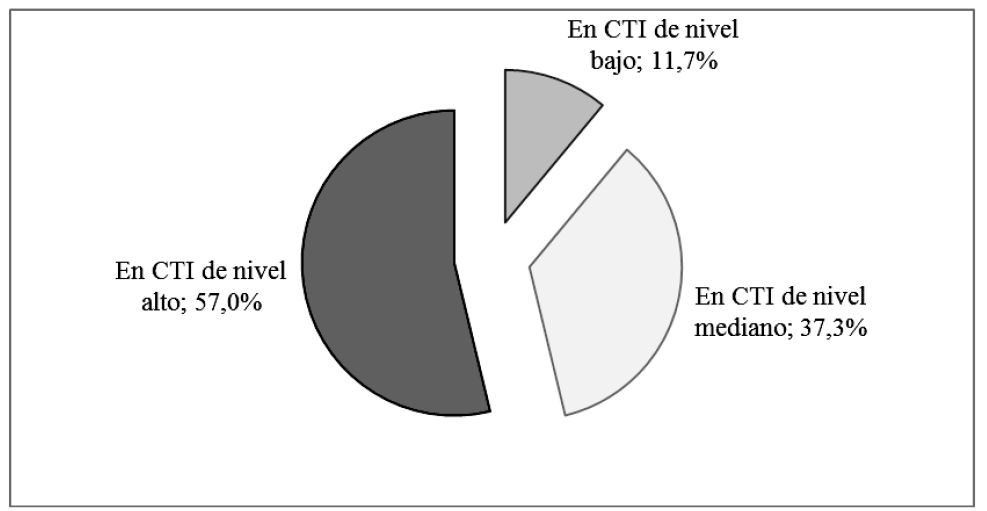

Fuente: Elaboración de los autores. Encuesta de Corporaciones Multinacionales, Proyecto COLEF-CONACYT \# 55108, "Firmas Multinacionales en México: un estudio sobre la estructura organizacional, la innovación y las prácticas de empleo", 2008-2009.

Lógicamente, con la información disponible hasta aquí, resulta difícil precisar si el nivel de CTI constituye una precondición a favor de la localización, o al revés, si es la actividad conjunta de las EMN lo que estaría desencadenando ambientes de CTI de niveles más elevados. Esta cuestión se tratará de puntualizar en el siguiente epígrafe.

\section{Los tipos de empresas según grado de éxito [en el eje combinado de innovación e inclusión] y el contexto innovador}

Una vez establecido el nivel de CTI asociado a los territorios donde operan las EMN, se puede volver a plantear la cuestión de qué tan relacionado está el grado de éxito en innovación e inclusión de las EMN con respecto al nivel de CTI de las entidades donde aquellas operan. El supuesto aquí sería que un entorno propicio de CTI favorecería la propensión de 
las EMN a innovar; y que este efecto, a su vez, se convierte en antecedente que auspiciaría actitudes y prácticas de inclusión. El resultado del cruce de estas dos variables se presenta en la gráfica 2, que muestra cómo los tipos de EMN se integran a cada uno de los ecosistemas de innovación.

Gráfico 2. Tipo de empresa multinacional, de acuerdo al nivel de CTI de la entidad donde radica la EMN en México

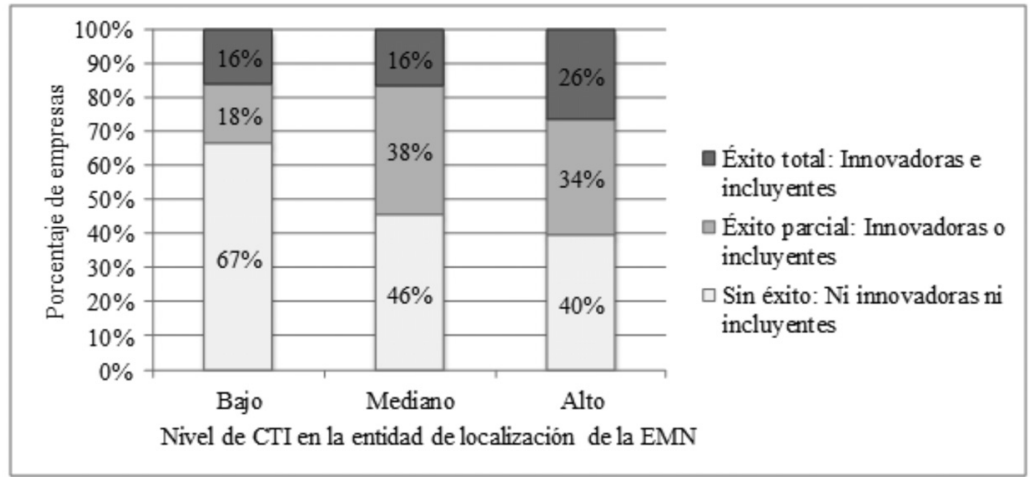

Fuente: Elaboración de los autores. Encuesta de Corporaciones Multinacionales, Proyecto COLEF-CONACYT \# 55108, "Firmas Multinacionales en México: un estudio sobre la estructura organizacional, la innovación y las prácticas de empleo", 2008-2009.

Lo que indica con claridad la gráfica es muy significativo, aunque nada sorprendente, por lo que hasta ahora se ha visto. Conforme a lo esperado, vemos que: (a) el porcentaje mayor de EMN en todos los ecosistemas corresponde a aquellas "sin éxito" - aunque sólo en los ecosistemas bajos es mayoría - y la proporción más pequeña, también en todos los ecosistemas, corresponde a EMN con "éxito total"; (b) existe una relación directa y consistente entre las dos variables. En efecto, a medida que aumenta el nivel de CTI en las entidades, al mismo tiempo, por un lado, va disminuyendo gradualmente la proporción de las empresas "sin 
éxito" en innovación e inclusión y, por el otro, va aumentando tendencialmente la proporción de empresas con "algún éxito (parcial o total)".

Casi está de más decir, y sólo se hace para reforzar una idea que resulta ostensible visualmente en la gráfica, que la prueba de Chi Cuadrada correspondiente a este cruce de variables resultó estadísticamente significativa, respaldando técnicamente la existencia de una relación entre ellas con un nivel de confianza de casi el 100\% ( $p$-value $=0.00$ ).

Ahora bien, más allá de conocer que las dos variables se encuentran estrechamente relacionadas, se precisaría determinar cuál es específicamente la naturaleza de la relación que guardan entre sí. Regresamos a la pregunta formulada antes: ¿son las características de los ecosistemas los que producen ciertos tipos de empresas o, por el contrario, son ciertos tipos de empresas, mediante su localización en determinados territorios, los que crean ecosistemas con características vinculantes?

Aunque ya se había sugerido que los tipos de empresas eran resultados dependientes de los contextos territoriales en los que se encontraban las EMN, la realidad es que, respecto a esta cuestión, aquí cabrían dos hipótesis alternativas. En una, los procesos de innovación e inclusión pueden considerarse como de naturaleza ex-post, en tanto se explicarían por el influjo diferencial de los distintos ecosistemas sobre las propiedades dinámicas de las empresas, entre las que estarían aquellas dos. Serían propiedades desarrolladas a posteriori, y deberían ser analizadas como un problema de evolución y transformación. El principio de la otra hipótesis es de carácter ex-ante. Las características de las empresas, a las que se ligarían inclinaciones y proclividades, son preexistentes a la localización. El establecimiento acumulado de empresas de cierto tipo en un territorio sería, en este caso, la clave para explicar las características de los ecosistemas de CTI. 
A partir de la información disponible en la base resulta difícil determinar con total certeza cuál de las dos hipótesis se encuentra más cercana a la realidad. Como se mencionó en la parte metodológica, los datos constituyen una visión de corte del momento en el que se levantó la encuesta. Pero en la medida en que ambas hipótesis suponen la existencia de procesos, con estados de inicio y de llegada diferentes, para dar cuenta de ello y poder responder cabalmente a la interrogante, se necesitarían series de datos. Sin embargo, aún cuando no sea posible, por esta razón, presentar todavía conclusiones definitivas, la información recogida en el cuadro 3 nos permite no obstante avanzar algo en dirección a la contrastación de las hipótesis, a través de la incorporación de la variable antigüedad a la ecuación.

Siguiendo la misma lógica que con otras variables utilizadas en este trabajo, la antigüedad también se redujo a dos categorías, antiguas y recientes, tomando como punto de corte el valor promedio del tiempo en años transcurrido desde el inicio de operaciones, en este caso 28 años. Así, las EMN que se establecieron antes de 1986 son consideradas antiguas para este análisis. $Y$ recientes, por su parte, serían entonces aquellas que lo hicieron después de esa fecha. También, para hacer más manejable el análisis, se hicieron igualmente dicotómicas tanto el grado de éxito en innovación e inclusión como el nivel de CTI en la entidad. Todos los porcentajes son sobre el total de empresas. 
Cuadro 3. Distribución de las EMN en los ecosistemas de innovación, según su antigüedad y tipo

\begin{tabular}{|c|c|c|c|c|c|c|c|}
\hline \multirow{4}{*}{$\begin{array}{l}\text { Nivel de CTI } \\
\text { en la entidad }\end{array}$} & \multicolumn{6}{|c|}{ Antigüedad } & \multirow{4}{*}{ Total } \\
\hline & & & \multirow{3}{*}{ Total } & \multicolumn{2}{|c|}{ Antigua } & \multirow{3}{*}{ Total } & \\
\hline & \multicolumn{2}{|c|}{$\begin{array}{l}\text { Éxito en innovación e } \\
\text { inclusión }\end{array}$} & & \multicolumn{2}{|c|}{$\begin{array}{c}\text { Éxito en innovación } \\
\text { e inclusión }\end{array}$} & & \\
\hline & $\begin{array}{l}\text { Total o } \\
\text { parcial }\end{array}$ & Sin éxito & & $\begin{array}{l}\text { Total o } \\
\text { parcial }\end{array}$ & Sin éxito & & \\
\hline Alto & $20.1 \%$ & $14.8 \%$ & $34.9 \%$ & $10.6 \%$ & $5.6 \%$ & $16.1 \%$ & $51 \%$ \\
\hline Medio/Bajo & $12.1 \%$ & $21.4 \%$ & $33.5 \%$ & $12.0 \%$ & $3.5 \%$ & $15.5 \%$ & $49 \%$ \\
\hline Total & $32.2 \%$ & $36.1 \%$ & $68.4 \%$ & $22.6 \%$ & $9.1 \%$ & $31.6 \%$ & $100 \%$ \\
\hline
\end{tabular}

Fuente: Elaboración de los autores. Encuesta de Corporaciones Multinacionales, Proyecto COLEF-CONACYT \# 55108, "Firmas Multinacionales en México: un estudio sobre la estructura organizacional, la innovación y las prácticas de empleo", 2008-2009.

A continuación, se presentan las ideas más relevantes que se pueden extraer del cuadro en relación con el examen de las dos hipótesis presentadas:

1. Más del doble de las EMN más antiguas son "exitosas" parcial o totalmente en innovación e inclusión frente a las "sin éxito" (22.6\% vs $9.1 \%)$. Inversamente, la mayoría de las recientes no es exitosa $(36.1 \%$ vs $32.2 \%$ ). Esto podría estar sugiriendo que alcanzar el "éxito" en innovación e inclusión es un proceso evolutivo de menos a más y, en ese sentido, estar apoyando la hipótesis ex-post.

2. Entre las EMN más antiguas, aquellas "sin éxito" actual en innovación e inclusión tienden a concentrarse en los CTI altos (5.6\% vs 3.5\%). Y, por el contrario, aquellas EMN que actualmente exhiben "éxito total o parcial" se localizan preferentemente en CTI más bajos (12 vs 10.6). Este resultado pareciera contradecir no solamente ambas hipótesis, sino también la relación general entre ambas variables examinada antes. Sin 
embargo, también cabría la idea de que la localización de las EMN no seguía antes los mismos criterios que en la actualidad. En este sentido, no niega la hipótesis ex-ante.

3. Entre las más recientes, la situación se invierte. Casi el doble de las EMN con "éxito total o parcial" en innovación e inclusión se localizan en CTI altos (20.1\% vs $12.1 \%)$. Aquellas "sin éxito", por su parte, lo hacen en su mayoría en CTI más bajos (21.4\% vs 14.8\%). Reforzando la conjetura anterior, la interpretación aquí puede ir en el sentido de que, en la actualidad, existe una mayor selectividad. Ya sea porque las EMN con propensión a la innovación y a la inclusión prefieren por sí mismas localizarse en entidades competentes, o porque, en parte, lo que caracterizaría a este tipo de entidades es promover activamente la atracción de cierto tipo de empresas en detrimento de otros. Tal idea también sería congruente con la hipótesis ex-ante.

En resumen, la información favorece sobre todo a la hipótesis ex-ante, en el sentido de que las EMN llevan desde el comienzo, como rasgo constitutivo, la semilla de la innovación y la inclusión, más que a la hipótesis alternativa en el sentido de que estas propiedades se desarrollarían ulteriormente como resultados de las influencias del contexto. Tanto las EMN ahora "exitosas" que antes de 1986 se localizaban en entidades con CTI bajos, como las antiguas actualmente "sin éxito" que se localizaron en CTI altos, parecen desafiar el contexto en el que han operado a lo largo de todos estos años. La condición actual en todas ellas contraviene lo que se esperaría que llegaría a suceder de ser válida la hipótesis ex-post. Pareciera ser entonces principalmente un asunto de selectividad en la localización de las EMN, una selectividad que podría ser doble, y estar comandando la relación entre las dos variables principales que se han venido analizando ("grado de éxito..." y "nivel de CTI..." ), correspondientemente en dos direcciones: (a) las EMN buscan localizarse en contextos 
de CTI afines a sus características, y; (b) las propias entidades, a la vez que buscan atraer EMN afines, no ofrecen facilidades a las que no lo son.

\section{Conclusiones}

Es generalizado el reconocimiento que se le otorga a la innovación como factor que cumple un servicio inequívoco en favor del crecimiento económico, de los aumentos de productividad y de la competitividad, tanto a nivel de las empresas como de las regiones y de los propios países. Pero es menos conocida la relación que tienen los procesos innovadores de las empresas con la inclusión, igualmente en función de la disminución de las desigualdades sociales y en apoyo a las diversidades humanas.

Los datos, como se planteó, presentan limitaciones. Las conclusiones por eso no pueden ser sino preliminares. De acuerdo a los resultados globales del estudio, basados en la encuesta a EMN, se pueden resumir las siguientes ideas a manera de conclusión:

1. En general hay más inclusión social que innovación, si bien estamos partiendo de un concepto de inclusión muy específico. Esto llama la atención debido a que la innovación está mucho más asociada con el valor agregado que la inclusión. Pero, por otro lado, ante la falta de salarios altos, estas políticas son importantes, no sólo por la imagen que transmite sobre la firma y que contribuye a su posicionamiento en el mercado, sino por la posible lealtad de los trabajadores. Aunque, de cualquier manera, lo más común es que las EMN carezcan de ambas condiciones.

2. Los datos no permiten determinar de manera indiscutible el sentido de la relación. Pero los resultados parecen apuntar a que es más probable que la propensión a innovar contribuya a prácticas inclusivas que al revés. 
3. La innovación con inclusión social es todavía una aspiración que cada vez más se plasma en discursos, políticas y agendas públicas tanto en México como en otros países de nivel intermedio, pero más como una meta que como una estrategia precisa para llegar hasta ella. En la realidad, las que manifiestan las dos condiciones constituyen una exigua minoría.

4. Si bien el entorno innovador pudiera ejercer alguna influencia positiva sobre estos procesos, tal y como lo marca la teoría, los resultados no parecen favorecer esta idea. Apoyan más la hipótesis de que la semilla de la inclusión y la innovación es un desarrollo endógeno de las empresas, lo que tiene lógica especialmente en un entorno institucional que no impone formas de inclusión en un sentido más amplio. Este sería el caso de la ausencia de políticas salariales activas y la falta de apoyo gubernamental a la formación y consolidación de actores colectivos con los recursos de poder suficientes para contrarrestar las inercias conservadoras y excluyentes que predominan en gran parte de las EMNs.

Jorge Hector Carrillo Viveros, Doctor en Sociología, es Director del Departamento de Estudios Sociales de El Colegio de la Frontera Norte y Miembro del Sistema Nacional de Investigadores Nivel 3, Tijuana, México.】carrillo@colef.mx

Redi Gomis Hernández, Doctor en Ciencias Sociales, Investigador del Departamento de Estudios Sociales en El Colegio de la Frontera Norte, Tijuana, México. $\measuredangle$ rgomis@colef.mx

Graciela Bensusán, Doctora en Ciencia Política, es profesora- investigadora en la Universidad Autónoma Metropolitana, Unidad Xochimilco, División de Ciencias Sociales y Humanidades, México.\bensusan@servidor.unam.mx 
Sociologias, Porto Alegre, ano 19, no 46, set/dez 2017, p. 198-227

\section{Referencias}

1. AYDALOT, Philippe. Milieux innovateurs en Europe. Paris: GREMI, 1986. $361 \mathrm{p}$.

2. BOCK, Lazlo. Getting to Work on Diversity at Google. Google Official Blog. Recuperado a partir de: https://googleblog.blogspot.com/2014/05/getting-to-work-on-diversity-at-google.html.

3. CARRILLO, Jorge. La importancia de las multinacionales en la sociedad global. Viejos y nuevos retos para México. Tijuana: El Colegio de la Frontera Norte y Juan Pablos Editor, 2012. 291 p.

4. CARRILLO, Jorge. Firmas Multinacionales en México: Un estudio sobre la estructura organizacional, la innovación y las prácticas de empleo [Resumen Ejecutivo]. Tijuana: El Colegio de la Frontera Norte, 2013. 71 p.

5. CARRILLO, Jorge; GOMIS, Redi. Empresas multinacionales en México: ¿innovación con inclusión social? En: FOXLEY, Alejandro; STALLINGS, Barbara (Eds.), Economías latinoamericanas. Cómo avanzar más allá del ingreso medio. Santiago de Chile: Corporación de Estudios para Latinoamérica (Cieplan) / Center for Latin American \& Latino Studies, American University, 2014. p. 391-427. .

6. COOKE, Philip; MORGAN, Kevin. The Intelligent Region: Industrial and Institutional Innovation in Emilia-Romagna. Cardiff: Regional Industrial Research, 1991. 83 p.

7. CYPHER, James; DIETZ, James. The process of economic development. 3 ed. Londres: Routledge, 2009. 597 p.

8. DRUCKER, Peter. Innovation and entrepreneurship: practice and principles. Nueva York: Harper Business, 1993. 77 p.

9. DUNNING, John. Multinational Enterprises and the Global Economy. Wokingham: Addison-Wesley Publishing Company, 1993, 687 p.

10. DUNNING, John; LUNDAN, Sarianna. Multinational Enterprises and the Global Economy. 2 ed. Cheltenham: Edward Elgar, 2008. 920 p.

11. DUTRÉNIT, Gabriela; ZÚÑIGA, Patricia. Ranking Nacional de Ciencia, Tecnología e Innovación. Capacidades y oportunidades de los Sistemas Estatales de CTI: Ranking 2013. Ciudad de México: Foro Consultivo Científico y Tecnológico (FCCYT), 2013, 207 p.

12. FLORIDA, Richard. Toward the Learning Region. Futures, v. 27, n. 5, p. 52736, 1995. 
13. FOSTER, Christopher; HEEKS, Richard. Conceptualising Inclusive Innovation: Modifying Systems of Innovation Frameworks to Understand Diffusion of New Technology to Low-Income Consumers. The European Journal of Development Research, v. 25, n. 3, p. 333-55. 2013.

14. GILL, Indermit; KHARAS, Homi. An East Asian Renaissance: Ideas for Economic Growth. Washington: The World Bank. 2007. 343 p.

15. KRISTENSEN, Peer; ZEITLIN, Jonathan. Local players in global games: the strategic constitution of a multinational corporation. Oxford: Oxford University Press. 2005. 374 p.

16. LEE, Keun. Modernización industrial y capacidad de innovación para un crecimiento incluyente: caso del este de Asia y sus enseñanzas. En: FOXLEY, Alejandro; STALLINGS, Barbara (Eds.), Economías latinoamericanas. Cómo avanzar más allá del ingreso medio. Santiago de Chile: Corporación de Estudios para Latinoamérica (Cieplan) / Center for Latin American \& Latino Studies, American University, 2014. p. 215-63.

17. OECD. Innovation and Inclusive Development (Report). Cape Town, South Africa: Organisation for Economic Co-operation and Development (OECD). 2013a. Recuperado a partir de: http://www.oecd.org/sti/inno/oecd-inclusive-innovation.pdf.

18. OECD. Líneas Directrices de la OCDE para Empresas Multinacionales [Revision 2011]. OECD Publishin. 2013b. 99 p.

19. OIT. Declaración tripartita de principios sobre las empresas multinacionales y la política social. 4 ed. Ginebra: OIT (Organización Internacional del Trabajo). 2006. 28 p. Recuperado a partir de: http://www.ilo.org/empent/Publications/WCMS_124924/lang--es/index.htm

20. OIT. Empresa inclusiva: guía para la contratación de personas con discapacidad. Buenos Aires: OIT. 2015. 66 p. Recuperado a partir de http://www.ilo. org/wcmsp5/groups/public/---americas/---ro-lima/---ilo-buenos_aires/documents/ publication/wcms_452866.pdf

21. PAGE, Scott. Making the Difference: Applying a Logic of Diversity. The Academy of Management Perspectives, v. 21, n. 4, p. 6-20. 2007.

22. PAPAIOANNOU, Theo. How inclusive can innovation and development be in the twenty-first century? Innovation and Development, v. 4, n. 2, p. 187-202. 2014. 